\title{
A Solution to the Similarity Registration Problem of Volumetric Shapes
}

\author{
Wanmu Liu ${ }^{1}$, Sasan Mahmoodi ${ }^{1}$, Michael J. Bennett ${ }^{2}$, and Tom Havelock ${ }^{2,3}$ \\ 1 School of Electronics and Computer Science, University of Southampton, UK \\ $\{$ w13g10, sm3\}@ecs.soton.ac.uk \\ 2 Southampton NIHR Respiratory Biomedical Research Unit, Southampton \\ University Hospital NHS Foundation Trust, UK \\ \{michael.bennett, T.Havelock\}@soton.ac.uk \\ 3 Faculty of Medicine, University of Southampton, UK
}

\begin{abstract}
This paper provides a novel solution to the volumetric similarity registration problem usually encountered in statistical study of shapes and shape-based image segmentation. Here, shapes are implicitly represented by characteristic functions (CFs). By mapping shapes to a spherical coordinate system, shapes to be registered are projected to unit spheres and thus, rotation and scale parameters can be conveniently calculated. Translation parameter is computed using standard phase correlation technique. The method goes through intensive tests and is shown to be fast, robust to noise and initial poses, and suitable for a variety of similarity registration problems including shapes with complex structures and various topologies.
\end{abstract}

Keywords: similarity registration, volumetric shapes, characteristic functions, registration of lungs.

\section{Introduction}

Similarity registration is a significant technique that handles isometric scale, rotation and translation in computer vision. Major applications of this technique are two folds: one is statistical study of shapes and the other is shape-based image segmentation. Vast amount of research about this topic has been done in 2-D, including [1 -4]. These works handle similarity registration using gradient methods that iteratively optimize similarity measures of shapes represented by SDFs (signed distance functions) [5]. In 2-D, the computational cost of these methods is generally acceptable, however, they are not feasible in similarity registration of volumetric shapes which often appear in medical data such as CT-scans, MRI and ultrasound images.

One method to be noted is the renowned ICP (iterative closed point) method proposed in [6]. It is a general solution to 3-D rigid registration (concerning only rotation and translation). Other popular $3-\mathrm{D}$ registration methods in the literature are frequently designed for range data or open surfaces (e.g. see [7, 8]) which are not related to the algorithm to be introduced in this paper.

G. Bebis et al. (Eds.): ISVC 2013, Part I, LNCS 8033, pp. 343-352, 2013.

(C) Springer-Verlag Berlin Heidelberg 2013 
The problem to be solved in this paper is the registration of two intact volumetric shapes.

The algorithm proposed here is an extension of the method proposed in [9] for 3-D shapes. However, rather than using SDFs, which are convenient representations in solving PDEs (partial differential equations), we prefer CFs (characteristic functions) which are concise representations of volumetric shapes. This algorithm calculates optimal shape scale, rotation, and translation without handling PDEs and is shown to be robust to noise and initial poses, and appropriate for many registration problems involving shapes with topological complexities mostly observed in medical data.

The rest of the paper is organized as follows. Section 2 presents preliminaries concerning shape registration. Section 3 describes the theory behind the registration technique proposed here. Numerical results are presented in section 4 and finally we conclude the paper in section 5 .

\section{Mathematical Preliminaries and Statement of the Problem}

In this paper, unit quaternions are used as mathematical representation of rotation of volumetric rigid shapes. A unit quaternion is a four vector $\boldsymbol{q}=$ $\left(q_{0}, q_{1}, q_{2}, q_{3}\right)$ with $q_{0}^{2}+q_{1}^{2}+q_{2}^{2}+q_{3}^{2}=1$. In our case, a unit quaternion consists of an axis, denoted by a unit vector $\mathbf{v}=\left(v_{1}, v_{2}, v_{3}\right)^{\mathrm{T}}$ and an angle denoted by $\Delta \theta$, making $\boldsymbol{q}(\mathbf{v}, \Delta \theta)=\left(\cos (\Delta \theta / 2), \mathbf{v}^{\mathrm{T}} \sin (\Delta \theta / 2)\right)[10]$. Unit vector $\mathbf{v}$ and angle $\Delta \theta$ are considered as the axis and angle of rotation. This is a linear transform and equivalent to a rotation generated by the corresponding $3 \times 3$ rotation matrix $\mathbf{R}(\boldsymbol{q}(\mathbf{v}, \Delta \theta))[10]$.

Let $\Omega \subset \mathbb{R}^{3}$ be bounded and represent the image domain, and $\Phi_{r}(\mathbf{x}): \Omega \rightarrow \mathbb{R}$ and $\Phi_{t}(\mathbf{x}): \Omega \rightarrow \mathbb{R}$ denote the characteristic functions (CFs) of reference shapes and target shapes. These functions are defined as

$$
\Phi(\mathbf{x})= \begin{cases}1, & \mathbf{x} \in \Omega_{+}, \\ 0, & \mathbf{x} \in \Omega_{-},\end{cases}
$$

where $\Omega_{+}$and $\Omega_{-}$respectively represent domains inside and outside shapes. The surfaces of shapes are implicit and of less importance in this work.

Let parameter $s$ represent scaling, $\boldsymbol{q}$ rotation and $\boldsymbol{T}=\left(T_{x}, T_{y}, T_{z}\right)^{\mathrm{T}}$ translation. The problem becomes to find a set of $s, \boldsymbol{q}$ and $\boldsymbol{T}$ that maximize the normalized inner product of shapes' CFs

$$
E_{R}=\int_{\Omega} \frac{\Phi_{t}(\mathbf{x}) \Phi_{r}(s \mathbf{R}(\boldsymbol{q}) \mathbf{x}-\boldsymbol{T})}{\left\|\Phi_{t}(\mathbf{x})\right\|\left\|\Phi_{r}(s \mathbf{R}(\boldsymbol{q}) \mathbf{x}-\boldsymbol{T})\right\|} \mathrm{d} \mathbf{x}
$$

where $\|\cdot\|$ is the $\mathbf{L}^{2}$-norm of CFs $\left(\|\Phi\|=\left(\int_{\Omega}|\Phi|^{2} \mathrm{~d} \mathbf{x}\right)^{\frac{1}{2}}\right)$.

In a geometric point of view, shapes are regarded as vectors and their similarity is measured by their normalized inner product. It is noted that $E_{R}$ is between 
0 and 1 , and similarity between shapes is intuitively measured by percentage. Using this measure, the maximum similarity that could be achieved between shapes is unity, which corresponds only to identical shapes.

\section{Method}

Before calculation of parameter, $\Phi_{r}(\mathbf{x})$ and $\Phi_{t}(\mathbf{x})$ are centralized, i.e.:

$$
\begin{gathered}
\breve{\Phi}_{r}(\mathbf{x})=\Phi_{r}\left(\mathbf{x}+\mathbf{c}_{r}\right), \\
\breve{\Phi}_{t}(\mathbf{x})=\Phi_{t}\left(\mathbf{x}+\mathbf{c}_{t}\right),
\end{gathered}
$$

where $\mathbf{c}_{r}$ and $\mathbf{c}_{t}$ are the respective centroids of both CFs calculated according to the initial Cartesian coordinate system.

For convenience calculating rotation and scale parameter, $\breve{\Phi}_{r}(\mathbf{x})$ and $\breve{\Phi}_{t}(\mathbf{x})$ are mapped to a spherical coordinate system. $\breve{\Phi}_{r}(\mathbf{x})$ and $\breve{\Phi}_{t}(\mathbf{x})$ are respectively represented by $\breve{\Phi}_{r}(\mathbf{r}): \Omega_{\mathcal{S}^{2}} \rightarrow \mathbb{R}$ and $\breve{\Phi}_{t}(\mathbf{r}): \Omega_{\mathcal{S}^{2}} \rightarrow \mathbb{R}$, where $\Omega_{\mathcal{S}^{2}} \subset \mathbb{R} \times \mathbb{S}^{2}$ denotes a spherical domain bounded with radius $R$ inside image domain $\Omega$ and $\mathbf{r}=(r, \hat{\mathbf{x}}(\theta, \varphi))^{\mathrm{T}}\left(\hat{\mathbf{x}}=(\cos (\theta) \sin (\varphi), \sin (\theta) \sin (\varphi), \cos (\varphi))^{\mathrm{T}}, r \in[0, R], \theta \in\right.$ $[0,2 \pi)$, and $\varphi \in[0, \pi])$.

This mapping allows the scale and rotation parameter to be separated, namely, to change from $\breve{\Phi}_{r}(s \mathbf{R} \mathbf{x})$ to $\breve{\Phi}_{r}(s r, \mathbf{R} \hat{\mathbf{x}})$, and these two parameters could be calculated individually.

\subsection{Rotation}

An optimal rotation could be represented by a unit quaternion $\boldsymbol{q}_{o p}$ which consists of a unit vector $\mathbf{v}_{o p}$ and an angle $\Delta \theta_{o p}$. Radial variable $r$ contains scale difference between the shapes to be registered, therefore to remove its impact on calculating rotation angle of the two $\mathrm{CFs}$, we integrate the $\mathrm{CFs}$ over variable $r$, i.e.:

$$
\begin{aligned}
& \tilde{\Phi}_{r}(\hat{\mathbf{x}}(\theta, \varphi))=\int_{0}^{R} \breve{\Phi}_{r}(\mathbf{r}) r^{2} \mathrm{~d} r \\
& \tilde{\Phi}_{t}(\hat{\mathbf{x}}(\theta, \varphi))=\int_{0}^{R} \breve{\Phi}_{t}(\mathbf{r}) r^{2} \mathrm{~d} r
\end{aligned}
$$

This indeed could be intuitively considered as projecting the CFs of shapes on to a parametric unit sphere centered by their centroids, referred to in this paper as $\mathcal{S}^{2}$ maps (see figure 1). The problem becomes to maximize the inner product of the two $\mathcal{S}^{2}$ maps:

$$
\boldsymbol{q}_{o p}=\underset{\boldsymbol{q}}{\operatorname{argmax}} \int_{\mathbb{S}^{2}} \tilde{\Phi}_{t}(\hat{\mathbf{x}}) \tilde{\Phi}_{r}(\mathbf{R}(\boldsymbol{q}) \hat{\mathbf{x}}) \mathrm{d} \hat{\mathbf{x}}
$$

To solve this equation, the unit sphere is mapped onto a bounded plane with coordinates $\dot{\theta}$ and $\dot{\varphi}$, referred to in this paper as $\mathcal{R}^{2}$ map. We prefer to generate an $\mathcal{R}^{2}$ map according to the axis around which the $\mathcal{S}^{2}$ map rotates, because 
in this way, rotation of an $\mathcal{S}^{2}$ map could be simply represented by shifting the

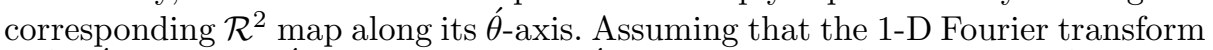
of $\tilde{\Phi}_{r}(\dot{\theta}, \dot{\varphi})$ and $\tilde{\Phi}_{t}(\dot{\theta}, \dot{\varphi})$ with respect to $\dot{\theta}$ are respectively $\tilde{\Psi}_{r}\left(\omega_{\dot{\theta}}, \dot{\varphi}\right)$ and $\tilde{\Psi}_{t}\left(\omega_{\dot{\theta}}, \dot{\varphi}\right)$, the optimal rotation angle by rotating around a fixed common axis $\mathbf{v}_{0}$ could be obtained by

$$
\Delta \theta_{o p}\left(\mathbf{v}_{0}\right)=\underset{\Delta \theta}{\operatorname{argmax}} \int_{0}^{\pi} \int_{-\infty}^{+\infty} \tilde{\Psi}_{t}\left(\omega_{\dot{\theta}}, \dot{\varphi}\right) \overline{\tilde{\Psi}_{r}\left(\omega_{\dot{\theta}}, \dot{\varphi}\right)} e^{i \omega_{\dot{\theta}} \Delta \theta} \sin (\dot{\varphi}) \mathrm{d} \omega_{\dot{\theta}} \mathrm{d} \dot{\varphi}
$$

The problem now is to find proper axes that could enable us to use the above equation. PCA is employed here to find these axes.

Using centralized CFs in section $3.2,3 \times 3$ symmetric covariance matrices $\Sigma_{r}$ and $\Sigma_{t}$ respectively for $\breve{\Phi}_{r}$ and $\breve{\Phi}_{t}$ could be computed as

$$
\Sigma=\frac{\int_{\Omega} \mathbf{x x}^{\mathrm{T}} \breve{\Phi}(\mathbf{x}) \mathrm{d} \mathbf{x}}{\int_{\Omega} \breve{\Phi}(\mathbf{x}) \mathrm{d} \mathbf{x}}
$$

We then calculate respective three eigenvectors of $\Sigma_{r}$ and $\Sigma_{t}$, denoted by $\mathbf{P}_{r}=$ $\left(\boldsymbol{p}_{r_{1}}, \boldsymbol{p}_{r_{2}}, \boldsymbol{p}_{r_{3}}\right)$ and $\mathbf{P}_{t}=\left(\boldsymbol{p}_{t_{1}}, \boldsymbol{p}_{t_{2}}, \boldsymbol{p}_{t_{3}}\right)$. The eigenvectors are ordered according to their eigenvalues, i.e. the first eigenvector corresponds to the largest eigenvalue. These three eigenvectors are referred to in this paper as the first, second and third principal axes. $\breve{\Phi}_{r}(\hat{\mathbf{x}})$ is rotated so that $\mathbf{P}_{r}$ is coincided with $\mathbf{P}_{t}$. This rotation is generated by $\boldsymbol{q}_{p}$, which is calculated by three steps:

Step 1: Calculating $\boldsymbol{q}_{p_{1}}$ that coincides the first principal axis,

$$
\begin{aligned}
& \Delta \theta_{p_{1}}=\cos ^{-1}\left(\boldsymbol{p}_{r_{1}} \cdot \boldsymbol{p}_{t_{1}}\right), \mathbf{v}_{p_{1}}=\left(\boldsymbol{p}_{r_{1}} \times \boldsymbol{p}_{t_{1}}\right) / \sin \left(\Delta \theta_{p_{1}}\right), \\
& \boldsymbol{q}_{p_{1}}=\left(\cos \left(\Delta \theta_{p_{1}} / 2\right), \mathbf{v}_{p_{1}}^{\mathrm{T}} \sin \left(\Delta \theta_{p_{1}} / 2\right)\right),
\end{aligned}
$$

Step 2: Calculating $\boldsymbol{q}_{p_{2}}$ that coincides the second principal axis,

$$
\begin{aligned}
& \Delta \theta_{p_{2}}=\cos ^{-1}\left(\mathbf{R}\left(\boldsymbol{q}_{p_{1}}\right) \boldsymbol{p}_{r_{2}} \cdot \boldsymbol{p}_{t_{2}}\right), \mathbf{v}_{p_{2}}=\left(\mathbf{R}\left(\boldsymbol{q}_{p_{1}}\right) \boldsymbol{p}_{r_{2}} \times \boldsymbol{p}_{t_{2}}\right) / \sin \left(\Delta \theta_{p_{2}}\right), \\
& \boldsymbol{q}_{p_{2}}=\left(\cos \left(\Delta \theta_{p_{2}} / 2\right), \mathbf{v}_{p_{2}}^{\mathrm{T}} \sin \left(\Delta \theta_{p_{2}} / 2\right)\right),
\end{aligned}
$$

Step 3: Calculating $\boldsymbol{q}_{p}$ by quaternion multiplication of $\boldsymbol{q}_{p_{1}}$ and $\boldsymbol{q}_{p_{1}}$,

$$
\boldsymbol{q}_{p}=\boldsymbol{q}_{p_{1}} \boldsymbol{q}_{p_{2}}
$$

Note that multiplication of quaternions follows principles in quaternion algebra and in certain cases the third principal axes may be inverse to each other after the coinciding, however, this would not affect the final result. Figure 2 presents a general process of coinciding shapes' principal axes. Then $\mathbf{P}_{t}$ is used as the axes according to which we apply equation 8 . There are three $\mathcal{R}^{2}$ maps generated according to the three coincided principal axes and consequently three rotation angles, $\Delta \theta_{t_{1}}, \Delta \theta_{t_{2}}$ and $\Delta \theta_{t_{3}}$ are calculated. With the principal axes and rotation angles, we have three quaternions, $\boldsymbol{q}_{t_{1}}, \boldsymbol{q}_{t_{2}}$ and $\boldsymbol{q}_{t_{3}}$. Finally, $\boldsymbol{q}_{o p}$ could be computed by

$$
\boldsymbol{q}_{o p}=\boldsymbol{q}_{p} \boldsymbol{q}_{t_{1}} \boldsymbol{q}_{t_{2}} \boldsymbol{q}_{t_{3}} .
$$




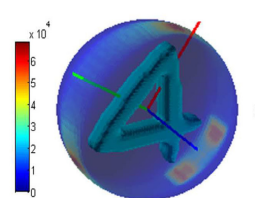

(a)

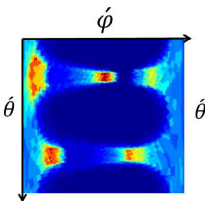

(b)

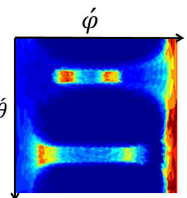

(c)

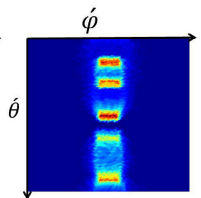

(d)

Fig. 1. Principal axes, $\mathcal{S}^{2}$ map and $\mathcal{R}^{2}$ maps of a volumetric '4'. (a) Red, green and blue lines are respectively the first, second and third principal axis. The sphere that contains the shape is its $\mathcal{S}^{2}$ map. (b)-(d) are the $\mathcal{R}^{2}$ maps acquired according to the first, second and third principal axis.

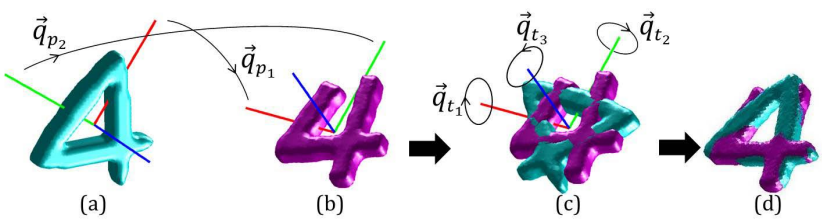

Fig. 2. (a) and (b) describe the process of coinciding the principal axes of shapes to be registered. (c) The following three adjustments according to the three principal axes of the reference shape. (d) The result after the adjustments.

\subsection{Scaling}

The scale parameter is computed according to the $\mathcal{S}^{2}$ maps discussed in the previous section. We start by considering a simple case that the reference shape is a rescaled version of the target shape:

$$
\breve{\Phi}_{t}(r, \hat{\mathbf{x}})=\breve{\Phi}_{r}(s r, \hat{\mathbf{x}})
$$

Integral over variable $r$ on the left side of the above equation is $\tilde{\Phi}_{r}$ (given in equation (6). Assuming that $r=s r$, integrals over variable $r$ on both sides therefore satisfy

$$
\begin{aligned}
\tilde{\Phi}_{t}(\hat{\mathbf{x}}) & =\int_{0}^{R} \breve{\Phi}_{r}(s r, \hat{\mathbf{x}}) r^{2} \mathrm{~d} r \\
& =\int_{0}^{R} \breve{\Phi}_{r}(\dot{r}, \hat{\mathbf{x}}) \frac{r^{2}}{s^{2}} \mathrm{~d} \frac{r}{s} \\
& =\frac{1}{s^{3}} \int_{0}^{R} \breve{\Phi}(\dot{r}, \hat{\mathbf{x}}) \dot{r}^{2} \mathrm{~d} \dot{r} \\
& =\frac{1}{s^{3}} \tilde{\Phi}_{r}(\hat{\mathbf{x}})
\end{aligned}
$$

In practice, the equality hardly holds because the shapes to be registered may well be different. Therefore, we simply minimize the SSD (sum of squared difference) between both sides to find an estimate of the scale parameter. Assuming that $s=1 / s^{3}$, the $\mathrm{SSD}$ could be written as 


$$
\begin{aligned}
E_{s} & =\int_{\mathbb{S}^{2}}\left|s \tilde{\Phi}_{r}(\hat{\mathbf{x}})-\tilde{\Phi}_{t}(\hat{\mathbf{x}})\right|^{2} \mathrm{~d} \hat{\mathbf{x}} \\
& =\left\|\tilde{\Phi}_{r}\right\|^{2} \dot{s}^{2}-2\left\langle\tilde{\Phi}_{t}(\hat{\mathbf{x}}), \tilde{\Phi}_{r}(\hat{\mathbf{x}})\right\rangle \dot{s}+\left\|\tilde{\Phi}_{t}\right\|^{2}
\end{aligned}
$$

This is a simple quadratic equation and the solution is

$$
s=\frac{\left\langle\tilde{\Phi}_{t}(\hat{\mathbf{x}}), \tilde{\Phi}_{r}(\hat{\mathbf{x}})\right\rangle}{\left\|\tilde{\Phi}_{r}\right\|^{2}}
$$

By substituting equation 14 to equation 13 , the minimum value of $E_{s}$ could be obtained by

$$
E_{\text {smin }}=-\frac{\left\langle\tilde{\Phi}_{t}(\hat{\mathbf{x}}), \tilde{\Phi}_{r}(\hat{\mathbf{x}})\right\rangle^{2}}{\left\|\tilde{\Phi}_{r}\right\|^{2}}+\left\|\tilde{\Phi}_{t}\right\|^{2}
$$

It could be observed from the above equation that $E_{s}$ is dependent only on the inner product of $\mathcal{S}^{2}$ maps $\left(\left\|\tilde{\Phi}_{r}\right\|^{2}\right.$ and $\left\|\tilde{\Phi}_{t}\right\|^{2}$ are constants) and it has been maximized in section 3.1. Finally, the optimal value of $s$ could be calculated using the following equation:

$$
s_{o p}=\left(\frac{\left\|\tilde{\Phi}_{r}\right\|^{2}}{\left\langle\tilde{\Phi}_{t}(\hat{\mathbf{x}}), \tilde{\Phi}_{r}\left(\mathbf{R}\left(\boldsymbol{q}_{o p}\right) \hat{\mathbf{x}}\right)\right\rangle}\right)^{\frac{1}{3}}
$$

\subsection{Translation}

Using the calculated optimal rotation and scale parameter, $\boldsymbol{q}_{o p}$ and $s_{o p}$, we obtain $\dot{\Phi}_{r}(\mathbf{x})=\Phi_{r}\left(s_{o p} \mathbf{R}\left(\boldsymbol{q}_{o p}\right) \mathbf{x}\right)$. Let $\boldsymbol{T}=\left(T_{x}, T_{y}, T_{z}\right)$ denote the translation parameter and the optimal translation parameter $\boldsymbol{T}_{o p}$ could be calculated by employing the method introduced in section 3.1

$$
\boldsymbol{T}_{o p}=\underset{\boldsymbol{T}}{\operatorname{argmax}} \int_{\mathbb{R}^{3}} \Psi_{t}(\boldsymbol{\omega}) \overline{\dot{\Psi}_{r}(\boldsymbol{\omega})} e^{i \boldsymbol{\omega} \cdot \boldsymbol{T}} \mathrm{d} \boldsymbol{\omega},
$$

where $\boldsymbol{\omega}=\left(\omega_{x}, \omega_{y}, \omega_{z}\right)$ is the spacial angular frequency. $\dot{\Psi}_{r}(\omega)$ and $\Psi_{t}(\omega)$ are respectively the $3 \mathrm{D}$ spacial Fourier transform of $\dot{\Phi}_{r}(\mathbf{x})$ and $\Phi_{t}(\mathbf{x})$.

\section{Experimental Results}

The method proposed here is implemented in MATLAB 7.11 on a PC station with a $2.67 \mathrm{GHz}$ Xeon processor and $12 \mathrm{~GB}$ RAM. Reference shapes are in cyan (light color), target shapes in magenta (dark color) and for visualization purpose, only the surface of the shapes are shown here. Some experimental data, including approximate shape size, execution time $t$ as well as the change of $E_{R}$ (in equation 2, normalized inner product as the similarity measure) before and after registration are presented in the captions of the corresponding figures. 


\subsection{Performance Analyses Using Complex Shapes}

Three performance analyses, namely, a noise and an initial pose test, and a comparison with the standard ICP method are presented here to provide evidence of the robustness of the algorithm. Relatively complex shapes with various topologies are used to carry out the analyses and they would focus mainly on rotation and scale accuracy, which are the major contribution of this work. In addition, the purpose of comparing the method proposed here to the ICP method is to address the local minima issue, which is the drawback of most gradient descent iterative optimization methods.

One example of registration using the method proposed here is given in figure 3. The reference shape is two linked symmetric rings and the target shape is similarly posed two asymmetric horseshoes. However, such topological variation has no impact on the technique proposed here. Keeping their poses fixed, a noise test is done by registering the linked rings to the horseshoes with manually added increasing level of randomly generated binary noise ('salt and pepper' noise) from $10 \%$ to $90 \%$ (9 levels in total). Figure 4 presents several examples of the horseshoes with different levels of noise.

The left side of figure 5 shows the result of the noise test. Each level of the noise is generated 10 times and is both inside and outside the shape to contaminate the region and the surface (the noise outside the shape goes no further than 3 voxels away from the surface). An apparent decrease of the mean of the normalized inner product between registered shapes can be observed, while the standard variation remains stable.

The accuracy is further measured by calculating the difference between the standard registration parameters computed without noise and those computed corresponding to the noise levels. For rotation which is represented by quaternions, the SSD is calculated and for scale, the absolute difference is computed. As is shown respectively in figure 5 middle and right, an increase of mean and variance can be observed as the level of the noise rises. However, it should be noted that the rotation error is under $0.1 \%$ and the scale error under $2 \%$. Therefore, it can be concluded that the algorithm proposed here is robust in presence of a significant amount of noise.

As for the initial pose test, the results are presented by figure 6. 744 initial poses (equally distributed in $S O(3)$ ) of the linked rings are chosen to register to the horseshoes with and without noise (the noise level selected in this test is maximum: 90\%). From the two curves in the figure 6 left, it can be observed that our algorithm is stable under these initial conditions. A same test is done using the ICP method to register the two shapes without noise and the result is showed in figure 6 right, there are $91 \%$ of the initial conditions that make the ICP method fall into local minima.

\subsection{Registration of Real-World Shapes}

Figure 7 presents a practical example for our method: registration of lungs. The structure of lungs is relatively complex including smooth contours and sharp 


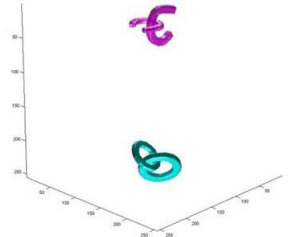

(a)

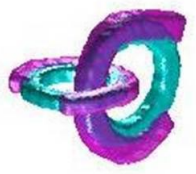

(b)

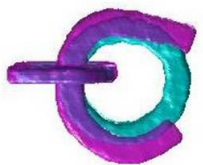

(c)

Fig. 3. The registration of regular and irregular shapes with different topologies. (a) The reference shape (two linked rings) and the target shape (two linked horseshoes). (b)The result of registration. (c)The result from another viewpoint. \{Reference shape size: $90 \times 60 \times 60$, target shape size: $90 \times 60 \times 60, t=1.75 \mathrm{~s}$, before: $E_{R}=0$, after: $E_{R}=0.54$. $\}$

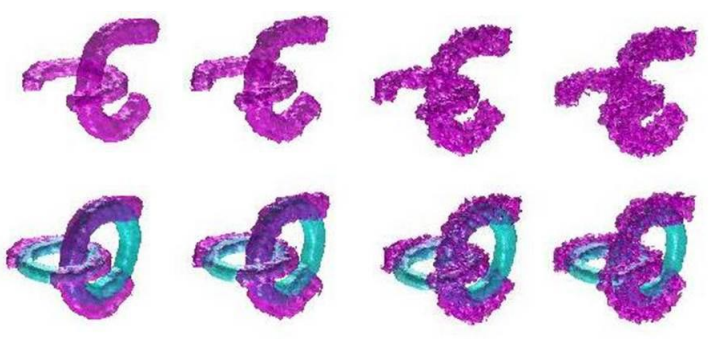

Fig. 4. Several examples of the horseshoes with various level of noise. Top row from left to right: $30 \%, 50 \%, 70 \%, 90 \%$. Bottom row from left to right: registration results corresponding to the noise levels.
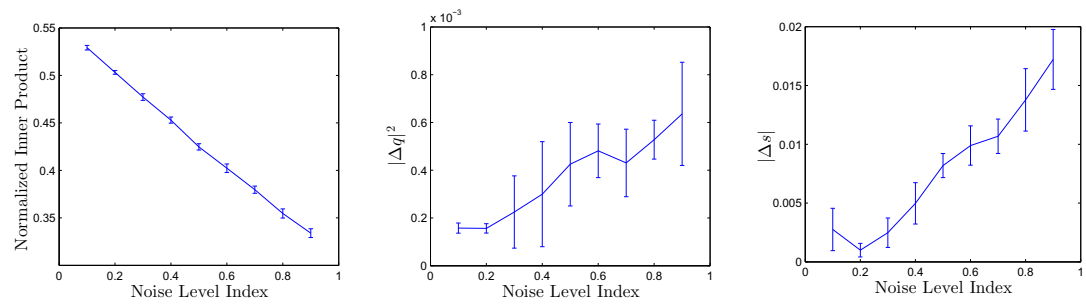

Fig. 5. A noise test. Left: the normalized inner products of shapes registered in presence of binary noise ('salt and pepper' noise) from $10 \%$ to $90 \%$. Middle: SSD between standard $\boldsymbol{q}$ calculated without noise and $\boldsymbol{q}$ with different levels of noise. Right: absolute difference between standard $s$ calculated without noise and $s$ with different levels of noise.

corners. It could be observed from figure 7 that our method achieves promising results and allows us to do further statistical studies. 

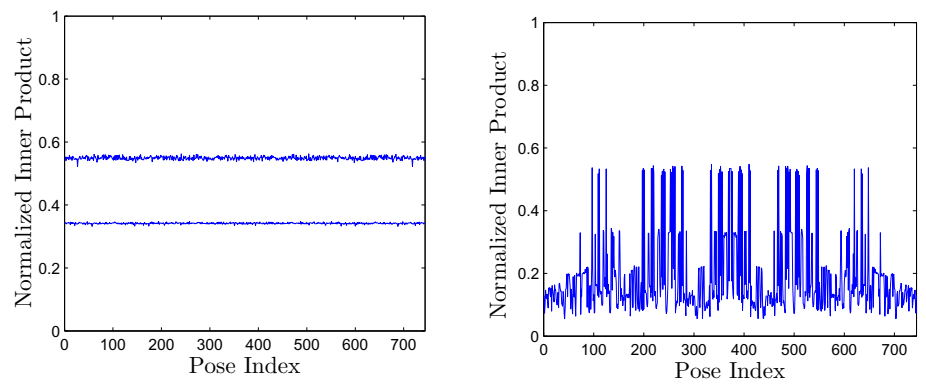

Fig. 6. An initial pose test. Left: the result using the method proposed here. The linked rings are registered to the horseshoes with noise (bottom curve) and without noise (top curve) under 744 initial conditions (equally distributed in $S O(3)$ ). Right: result of registration using the ICP method under 744 initial conditions (the horseshoes are without noise).

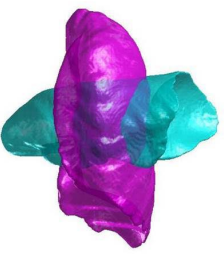

(a)

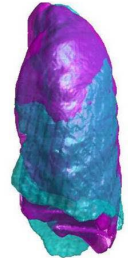

(b)

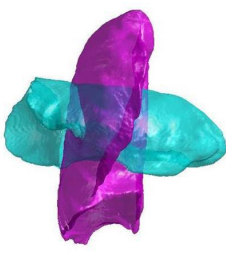

(c)

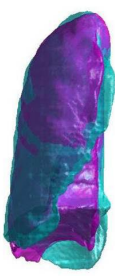

(d)

Fig. 7. The registration of lungs from two persons. (a) The reference and the target left lungs before registration. (b) The result of registration. \{Reference shape size: $150 \times 120 \times 270$, target shape size: $160 \times 120 \times 300, t=11.09 \mathrm{~s}$, before: $E_{R}=0.39$, after:

$E_{R}=0.85$. $\}$ (c) The reference and the target right lungs before registration. (d) The result of registration. $\{$ Reference shape size: $150 \times 110 \times 260$, target shape size: $160 \times$ $110 \times 270, t=9.47 \mathrm{~s}$, before: $E_{R}=0.32$, after: $E_{R}=0.83$. $\}$

\section{Conclusion}

This paper proposes a robust registration technique of two volumetric shapes represented by CFs. By mapping shapes to a spherical coordinate system, scale and rotation parameter could be handled separately. PCA is employed to find principal axes of shapes that largely facilitates the calculation of rotation parameter. Both computations of rotation and translation parameter exploit FFT, allowing the efficiency of our method to be vastly improved. The results of intensive experiments suggest that our method is able to register shapes with different topologies, and is robust to noise and initial conditions, and also efficient. It is a suitable solution to the registration problem in statistical modelling of volumetric shapes and shape-based volumetric image segmentation. 


\section{References}

1. Bresson, X., Vandergheynst, P., Thiran, J.: A variational model for object segmentation using boundary information and shape prior driven by the mumford-shah functional. International Journal of Computer Vision 68, 145-162 (2006)

2. Huang, X., Paragios, N., Metaxas, D.: Shape registration in implicit spaces using information theory and free form deformations. IEEE Transactions on Pattern Analysis and Machine Intelligence 28, 1303-1318 (2006)

3. Paragios, N., Rousson, M., Ramesh, V.: Non-rigid registration using distance functions. Computer Vision and Image Understanding 89, 142-165 (2003)

4. Chen, Y., Tagare, H., Thiruvenkadam, S., Huang, F., Wilson, D., Gopinath, K., Briggs, R., Geiser, E.: Using prior shapes in geometric active contours in a variational framework. International Journal of Computer Vision 50, 315-328 (2002)

5. Osher, S., Sethian, J.: Fronts propagating with curvature-dependent speed: algorithms based on hamilton-jacobi formulations. Journal of Computational Physics 79, 12-49 (1988)

6. Besl, P., McKay, N.: A method for registration of 3-d shapes. IEEE Transactions on Pattern Analysis and Machine Intelligence 14, 239-256 (1992)

7. Gelfand, N., Mitra, N., Guibas, L., Pottmann, H.: Robust global registration. In: Proceedings of the Third Eurographics Symposium on Geometry Processing, p. 197. Eurographics Association (2005)

8. Breitenreicher, D., Schnörr, C.: Robust 3d object registration without explicit correspondence using geometric integration. Machine Vision and Applications 21, 601$611(2010)$

9. Al-Huseiny, M., Mahmoodi, S., Nixon, M.: Robust rigid shape registration method using a level set formulation. In: International Symposium in Visual Computing, pp. 252-261 (2010)

10. Diebel, J.: Representing attitude: Euler angles, unit quaternions, and rotation vectors. Matrix (2006) 